\title{
SPERM TRANSPORT IN THE FEMALE RABBIT: THE RÔLE OF THE CERVIX
}

\author{
D. B. MORTON* AND T. D. GLOVER \\ Unit of Reproductive Biology, Life Sciences Building, University of Liverpool, \\ Crown Street, Liverpool L69 3BX
}

(Received 19th September 1973)

\begin{abstract}
Summary. Rabbit does were killed at intervals between 2 min and $90 \mathrm{hr}$ after mating and spermatozoa were flushed from the genital tract and counted. A technique is described which facilitates the counting of small numbers of spermatozoa with reasonable accuracy. Evidence was found to suggest that the rabbit's cervix functions as a sperm reservoir and factors influencing the formation of the reservoir and transcervical migration are discussed. It was noted that spermatozoa reached the oviduct within $30 \mathrm{~min}$ of coitus.
\end{abstract}

\section{INTRODUCTION}

In animals such as man and ruminants in which semen is mainly deposited in the vagina at coitus, the cervix appears to function as a sperm reservoir, i.e. spermatozoa migrate into the cervix (an environment more conducive to sperm survival than elsewhere in the female genital tract) and subsequently are slowly released into the uterus over a period of several hours (Nicholson, 1965; Mattner, 1966, 1968). By virtue of this constant flow of spermatozoa from the cervix, together with phagocytosis of spermatozoa within the tract and sperm passage into the abdominal cavity, it is envisaged that a population of 'fit' spermatozoa can be maintained in the oviduct, particularly at the site of fertilization. In species in which whole semen passes into the uterine horns at coitus, the uterotubal junction may act as a sperm reservoir, as in the pig (Rigby, 1964), or the uterine glands may serve this function, as probably occurs in the dog (Doak, Hall \& Dale, 1967).

Since insemination is intravaginal in the rabbit, it might be expected that in this species also the cervix would behave as a sperm reservoir following coitus, but clear evidence on this point is lacking. In the classic study of sperm transport by Braden (1953), the cervix was not considered as a separate entity and so it was the purpose of the present study to investigate sperm distribution after coitus, with particular reference to those regions of the female genital tract likely to act as sperm reservoirs.

\section{MATERIALS AND METHODS}

New Zealand White rabbits or a first cross with Californian rabbits were used.

* Present address: Strangeways' Research Laboratory, Wort's Causeway, Cambridge GB1 4RN. 
They were individually caged at constant temperature $\left(26^{\circ} \mathrm{C}\right.$, in a thermostatically maintained room) given a minimum of $12 \mathrm{hr}$ light every $24 \mathrm{hr}$ and were allowed free access to a diet of SGl (Nutrients Ltd, Liverpool) rabbit finisher pellets and water.

Oestrous does were obtained either by leaving them individually caged until they became receptive, or by inducing pseudopregnancy with an intravenous injection of 25 i.u. HCG ('Pregnyl', Organon Ltd). The does were then tested daily for oestrus from 2 weeks after the injection and mated two or three times with New Zealand White bucks. The artificially collected ejaculates regularly exhibited a high motility grading throughout the experimental period, which was mainly between July and September of one year. Does were killed by an overdose of sodium pentobarbitone $(70 \mathrm{mg} / \mathrm{kg}$ body wt given rapidly intravenously) and the interval between mating and euthanasia varied between $2 \mathrm{~min}$ and $90 \mathrm{hr}$. Immediately after death, artery forceps were used to divide the tract into vagina, cervix, caudal and cranial halves of the uterus, uterotubal junction (i.e. $1 \mathrm{~cm}$ of uterine horn and $1 \mathrm{~cm}$ of isthmus) and oviduct. Each segment of the tract was dissected free from fat, measured, separated from adjacent segments and flushed with $3 \mathrm{ml}$ of a $0.5 \%$ solution of 'Teepol' into plastic tubes, using a clean disposable syringe and needle on each occasion. The oviduct was flushed only once, but other segments were re-flushed four times with the original $3 \mathrm{ml}$ flushing fluid.

As vaginal fluid, usually containing millions of spermatozoa, bathes the external os of each cervix, the technique used to flush the cervix was slightly modified so that only those spermatozoa which had actually penetrated the cervical canal would be counted. The external os of each cervix was washed with $1 \mathrm{ml}$ of flushing solution (this was counted with the vaginal flushing) before flushing the cervical canal.

Instruments were thoroughly washed and soaked in chromic acid before use and the operator's hands were rinsed after each segment had been flushed. Areas likely to contain least spermatozoa were handled first and special care was taken to avoid contamination from the cut ends of the vagina when removing and dissecting out the tract.

\section{Sperm counting}

All counts were carried out using phase-contrast microscopy. Three methods of counting were used according to the number of spermatozoa counted in a preliminary haemocytometer slide (modified Fuchs-Rosenthal type) count. The main criterion was that at least 200 spermatozoa should always be counted whichever method was used.

Method 1. If the number of spermatozoa in the preliminary count exceeded fifteen per large square, the count was completed on a haemocytometer slide.

Method 2. If fewer than fifteen spermatozoa per large square but more than two in the entire grid were found, $0.4 \mathrm{ml}$ of the flushing was pipetted under a 22-mm square cover-slip mounted on a glass slide by four vaseline pillars. The coverslip was depressed so that fluid filled its area, and $30 \mathrm{~min}$ were allowed for the spermatozoa to settle out before counting. Evaporation from the sides of the counting chamber was prevented by placing it in a humid 
atmosphere until it was counted. Using either high- or low-power objectives of the microscope, traverses were made across the slide and the number of spermatozoa was counted. Two traverses were usually made, but if the sperm density was high, fractions of a traverse were counted. Equal areas were counted in diagonally opposite sides of the slide to allow for any variation in chamber depth. To calculate the number of spermatozoa present, the proportion of the total chamber area counted and also the proportion of flushing volume counted had to be considered.

Method 3. If less than two spermatozoa in the entire grid of the haemocytometer slide were initially counted, the flushing was evaporated to about $0.4 \mathrm{ml}$ (in a water bath at $50^{\circ} \mathrm{C}$ and blowing air over the surface of the flushing) and then counted as in Method 2. Using this method, the number of spermatozoa in the entire flushing could be counted if necessary.

An evaluation of the efficiency of the above methods has been described (Morton, 1970), and it was concluded that, using these methods, about $80 \%$ of spermatozoa in the tract could consistently be recovered.

\section{RESULTS}

Thirty-seven rabbits were used in the experiment. Except for $\frac{1}{2}, 1,2,3$ and $4 \mathrm{hr}$ when four, five, three, three and two rabbits, respectively, were killed, only one rabbit was killed at the other time intervals. The distribution of spermatozoa with time is shown in Text-fig. 1: the mean number of spermatozoa recovered from each region of the female genital tract (totalled for both sides of the tract) was plotted against time after mating. The logarithm $(x+1)$ of the sperm count was used in statistical calculations in order to normalize the data. This reduced the artificial weighting caused when exceptionally large numbers of spermatozoa were recovered from any one group. It can be seen that whereas the number of spermatozoa in the vagina rapidly decreased shortly after coitus, the number recovered from other regions increased, reached a peak and then gradually declined.

Artificial ejaculates were obtained from bucks throughout the experimental period and seminal analysis indicated that does had probably been inseminated with between 500 and $1500 \times 10^{6}$ spermatozoa. Only $23 \times 10^{6}$ spermatozoa, however, were recovered from the cranial vagina $4 \mathrm{hr}$ later. This drastic rate of depletion was not maintained, but was succeeded by a slower rate of decline. Maximum numbers of spermatozoa (i.e. about $10 \times 10^{6}$ ) were found in the cervix $2 \mathrm{hr}$ after mating and this peak was followed by a gradual decline over the next $72 \mathrm{hr}$ but spermatozoa could still be recovered from the cervix in fairly large numbers $\left(10^{4}\right)$ even at $90 \mathrm{hr}$.

In the caudal and cranial halves of the uterus, maximum numbers of spermatozoa (between 1 and $3 \times 10^{6}$ ) were recovered between 4 and $9 \frac{1}{2}$ hr post coitum; both these peaks were lower and also slightly later than the peak for the cervix. The rate of decline of sperm numbers in the uterus was more precipitate than in the cervix. A similar pattern to that for the cranial half of the uterus was evident for the uterotubal junction, except that fewer spermatozoa were recovered and maximum numbers $\left(3\right.$ to $6 \times 10^{5}$ ) were found slightly later, i.e. between $9 \frac{1}{2}$ and $12 \frac{1}{2} \mathrm{hr}$. 

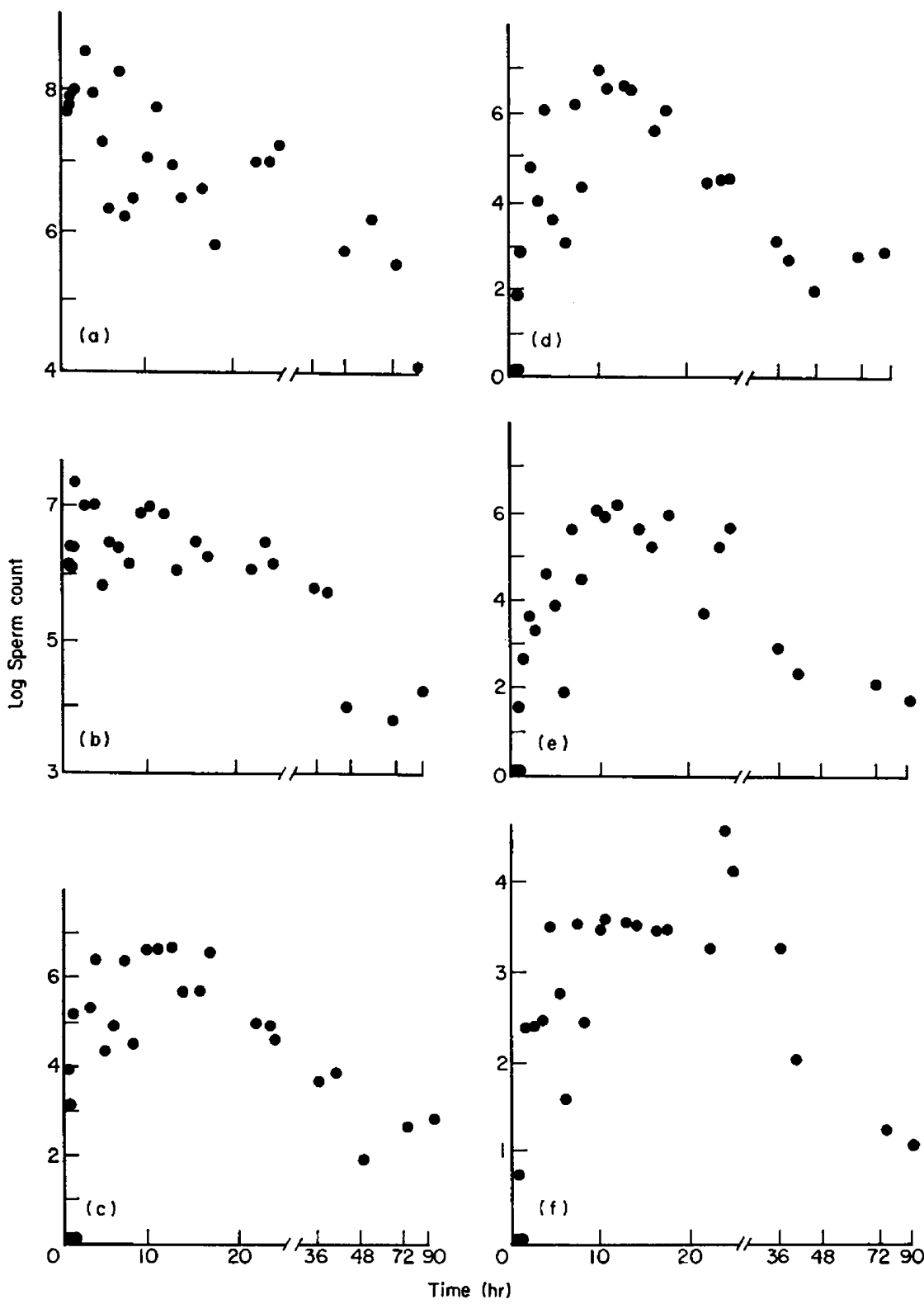

Texr-Fic. 1. The logarithm mean total number of spermatozoa recovered from both sides of a given region of the female genital tract from rabbits killed at various intervals after coitus. (a) Vagina; (b) cervix; (c) caudal uterus; (d) cranial uterus; (e) uterotubal junction; (f) oviduct. 
It has been found in other species that the uterotubal junction acts as a sperm reservoir but no evidence was found for this in the rabbit. Spermatozoa were first recovered from the oviduct $30 \mathrm{~min}$ after mating (in two out of four does) but maximum numbers were not consistently recovered until after $9 \frac{1}{2} \mathrm{hr}$. During the period of ovulation, between 1000 and 2000 spermatozoa were present in each oviduct and this level was maintained for another $24 \mathrm{hr}$ or so.

Highly significant positive correlations $(P<0.001)$ were found between all adjacent regions of the tract (Table 1) except for the correlation between the cervix and the caudal uterus, which was only significant at the $5 \%$ level.

Table 1. Correlation between the logarithm number of spermatozoa recovered from adjacent regions of the rabbit genital tract $(\log y=\mathrm{a}+\mathrm{b} \log x$ or arithmetically $\left.y=10^{\mathrm{a}} \cdot x^{\mathrm{b}}\right)$

\begin{tabular}{l|l|c|c}
\hline \multicolumn{1}{c|}{ Adjacent regions } & $\begin{array}{l}\text { Correlation } \\
\text { coefficient }\end{array}$ & $\begin{array}{c}\text { Regression } \\
\text { coefficient } \dagger \\
(b)\end{array}$ & $\begin{array}{c}\text { Intercept } \\
(a)\end{array}$ \\
\hline Vagina-cervix & $0.736^{* * *}$ & $0.348 \pm 0 \cdot 162$ & 2.582 \\
Cervix-caudal uterus & $0 \cdot 393^{*}$ & $1.453 \pm 1.218$ & -5.189 \\
Caudal uterus-cranial uterus & $0.924^{* * *}$ & $1 \cdot 022 \pm 0.123$ & -0.567 \\
Granial uterus-uterotubal & $0 \cdot 887^{* * *}$ & $0.788 \pm 0 \cdot 128$ & 0.390 \\
junction & $0.846^{* * *}$ & $0.575 \pm 0.130$ & 0.455 \\
\hline Uterotubal junction-oviduct & & \\
\hline
\end{tabular}

$\dagger$ Regression coefficients are given with $95 \%$ confidence limits.

* $P<0.05,{ }^{* * *} P<0.001$.

\section{DISCUSSION}

The evidence presented supports the hypothesis that the cervix of the female genital tract of the rabbit serves as a sperm reservoir. It was shown that spermatozoa could be recovered from the cervix in larger numbers and could persist there for longer periods of time than in either the uterus or oviduct. These parameters are even more pronounced when the results are expressed as sperm number per unit length of genital tract (the average length of the cervix, uterus and oviduct at autopsy was found to be $1.72 \mathrm{~cm}, 9.69 \mathrm{~cm}$ and $12.4 \mathrm{~cm}$, respectively). Detailed studies of the cervical sperm reservoir in ruminants have been carried out (Mattner, 1966, 1968) and three factors appear to contribute to the reservoir action of the cervix and these features may also be found in the rabbit. First, the mucosal folding of the rabbit's cervix is very complex (Hafez \& Kanagawa, 1972) and it can be appreciated that spermatozoa may well be trapped in this maze. Secondly, leucocytes were found to be far less numerous in the cervical flushings compared with those from the vagina or uterus (Morton, 1970); this suggests that less phagocytosis of spermatozoa takes place in the cervix. And thirdly, cervical mucus, helps to direct spermatozoa to the cervical crypts where the reservoir is formed (Mattner, 1968), but is said to be scant in the cervix of the rabbit during oestrus (Parker, 1931; Hamilton, 1951). Mucus is often found in the vagina, however, and is almost certainly of cervical origin. Even if the amount present within the cervical lumen is relatively small in volume, it may still be sufficient in relation to the size of the cervix to perform a function similar to that in the ruminant. As the cervix may 
well act as a sperm reservoir in the human (Nicholson, 1965), the rabbit could be a useful experimental model on which to assay contraceptive agents aimed at inhibiting the formation of the cervical reservoir.

Two observations from this study on the formation of the reservoir deserve comment: one is that so few spermatozoa enter the cervix despite the large number in the ejaculate. This can be explained by the expulsion of semen by does within minutes or even seconds of coitus; thus, if $500 \times 10^{6}$ spermatozoa were desposited during mating, only $20 \%$ could be recovered $30 \mathrm{~min}$ later. The second observation is that the number of spermatozoa recovered from the cervix reached a maximum $2 \mathrm{hr}$ after mating and subsequently declined, even though throughout the time period studied there were consistently far larger sperm numbers in the vagina than in the cervix. The reason for the early peak in the cervical count is most likely to be that vaginal secretions immobilize spermatozoa and they are then unable to migrate into the cervix. It has been shown that human vaginal secretions immobilize spermatozoa within 1 to $2 \mathrm{hr}$ of coitus (Weisman, 1939; Masters \& Johnson, 1966; Davajan, Nakamura \& Kharma, 1970) but little work has been carried out on this point in other species. Evidence for the passage of non-motile spermatozoa through the rabbit's cervix is equivocal although the balance indicates they are unable to do so (Noyes, Adams \& Walton, 1958; Morton, 1970), and comparable observations in sheep have shown that immotile spermatozoa do not penetrate deeply into the cervix (Mattner \& Braden, 1969; Lightfoot \& Restall, 1971). It seems reasonable to conclude that the continual discrepancy between cervical and vaginal counts is a sequel to sperm immobilization by the vaginal secretions.

The rapid elimination and immobilization of spermatozoa by the vagina would make it essential that they should progress to a more favourable environment quickly and this seems to be the case. Bedford (1971) has shown that $5 \mathrm{~min}$ after mating the vaginal pool is unnecessary since by this time nearly $1 \times 10^{6}$ spermatozoa have entered the cervical canal (Text-fig. 1 ).

The term 'cervical barrier' has come into use because of the large discrepancy between the number of spermatozoa recovered from the uterus and the number in an inseminate. Whilst accepting that the cervix does act as a mechanical obstruction, semen expulsion and sperm immobilization appear to be two other major reasons for the inefficient transport. A comparison of the uterine sperm count with the cervical count indicates (Table 2) that there is a relatively small barrier-like effect compared with the difference between the vagina and cervix (which is a measure of sperm immobilization), and the inseminate and the vagina (which is a measure of semen expulsion). This relatively small barrier-like action is probably synonymous with its function as a reservoir.

The positive correlations found between sperm counts in adjacent regions of the tract emphasize the dependence of counts in the more cranial parts of the tract on the caudal areas. The more spermatozoa that enter the cervical reservoir the more will reach the oviduct, increasing the chance of fertilization. In addition the larger the reservoir, the longer an adequate population of spermatozoa will be maintained in the oviduct. Spermatozoa may leave the cervix by their own motility or be passively transported by cervical and uterine contractions. 
Most work on sperm capacitation has involved preincubation in the uterus and the time needed for capacitation appears to be several hours (see review by Bedford, 1970). As uterine contractions probably propel spermatozoa through the uterus too quickly for them to capacitate fully there (Morton, 1970; Fuchs, 1972), an attempt was made to determine whether spermatozoa could undergo this stage of maturation in the cervix, where they are most likely to spend sufficient time. In a preliminary experiment, washed spermatozoa were preincubated in the isolated cervix for 4 and $8 \mathrm{hr}$. Spermatozoa were flushed from the cervix and injected $(0.05 \mathrm{ml}$ containing 80,000 spermatozoa) into the oviducts (isthmus) of four recipient does which had received an injection of 35 i.u. HCG $12 \frac{1}{2}$ to $13 \mathrm{hr}$ previously. Using cervical spermatozoa preincubated for $4 \mathrm{hr}$, two out of thirteen eggs cleaved whereas using spermatozoa preincubated for $8 \mathrm{hr}$, eleven out of twenty-two eggs cleaved. These results suggest that spermatozoa can complete the first stage of capacitation in the cervix.

Table 2. The difference between the number of spermatozoa recovered from vagina, cervix and uterus of the rabbit in the $4 \mathrm{hr}$ after mating, assuming that $500 \times 10^{6}$ spermatozoa were in the ejaculate

\begin{tabular}{c|c|c|c}
\hline $\begin{array}{c}\text { Time } \\
(\mathrm{hr})\end{array}$ & $\begin{array}{c}\text { Difference between } \\
\text { vaginal count and } \\
\text { ejaculate } \\
\left(\times 10^{6}\right)\end{array}$ & $\begin{array}{c}\text { Difference between } \\
\text { cervical and vaginal } \\
\text { counts } \\
\left(\times 10^{6}\right)\end{array}$ & $\begin{array}{c}\text { Difference between } \\
\text { uterine and cervical } \\
\text { counts } \\
\left(\times 10^{6}\right)\end{array}$ \\
\hline \multirow{1}{*}{} & 405 & 93 & $1 \cdot 7$ \\
1 & 385 & 113 & $1 \cdot 8$ \\
2 & 295 & 194 & $10 \cdot 8$ \\
3 & 409 & 83 & $8 \cdot 6$ \\
4 & 486 & 12 & $3 \cdot 2$ \\
& Seminal & Sperm & Cervical \\
& expulsion & immobilization & barrier' \\
\hline
\end{tabular}

Spermatozoa were first recovered from the oviducts 30 min after mating. This is earlier than previous workers have found (Skateine \& Rumiantzewa, 1948; Chang, 1952; Braden, 1953; Krehbiel, Lodge \& Sharma, 1972) and is probably due to the more sensitive sperm recovery technique used. This rapid transport correlates well with fertilization rates obtained after post-coital ligations (Adams, 1956; Greenwald, 1956). Both these workers found maximum ovum cleavage 3 to $4 \mathrm{hr}$ after mating, a time when large numbers of spermatozoa are present in the oviduct (see Text-fig. 1). Ligation of the oviduct at $\frac{1}{2}$ and $1 \frac{1}{4} \mathrm{hr}$, however, resulted in $0 \%$ and $1.6 \%$ of eggs being fertilized, respectively, and as spermatozoa have been found in the oviduct at these times (Textfig. 1), it would suggest that insufficient numbers had arrived or survived until ovulation.

It is interesting to note that the average rate of decline of the cervical count between 2 and $20 \mathrm{hr}$ post coitum is about 200,000 spermatozoa/hr and that between 7 and $24 \mathrm{hr}$, the numbers of spermatozoa in regions cranial to the cervix remain fairly constant. If retrograde passage of spermatozoa from the cervix into the vagina is considered to be negligible during this latter period, then about 200,000 spermatozoa would enter the uterus each hour. The uterine and oviducal sperm counts could be maintained at their respective levels by 
processes such as phagocytosis of spermatozoa and sperm passage into the abdominal cavity; sperm phagocytosis in the uterus is probably the major factor. It would be interesting to know the rate of change of the sperm population in these two regions of the genital tract; a rapid turnover of spermatozoa at the site of fertilization would help maintain a 'fit' population of male gametes for fertilization and this would be of particular benefit to spontaneous ovulators, in which ovulation may not occur for a relatively long period after mating.

\section{ACKNOWLEDGMENTS}

We wish to thank the Agricultural Research Council, the Population Council and the University of Liverpool for financial support. Thanks are also due to Mrs G. P. Boyd for technical assistance and to Mrs A Morrison for typing the manuscript.

\section{REFERENCES}

Adams, C.E. (1956) A study of fertilization in the rabbit: the effect of post-coital ligation of the Fallopian tube or uterine horn. F. Endocr. 13, 296.

BedFord, J. M. (1970) Sperm capacitation and fertilization in mammals. Biol. Reprod., Suppl. 2, 128.

BEDFoRd, J. M. (1971) The rate of sperm passage into the cervix after coitus in the rabbit. F. Reprod. Fert. 25, 211.

Braden, A. W. H. (1953) Distribution of sperms in the genital tract of the female rabbit after coitus. Aust. 7. biol. Sci. 6, 693.

Chang, M. G. (1952) Fertilizability of rabbit ova and the effects of temperature in vitro on their subsequent fertilization and activation in vivo. F. exp. Zool. 121, 351.

Davajan, N., Nakamura, R. M. \& Kharma, K. (1970) Spermatozoan transport in cervical mucus. Obstetl gynec. Surv. 25, 1.

DoAK, R. L., Hall, A. \& DALE, H. E. (1967) Longevity of spermatozoa in the reproductive tract of the bitch. F. Reprod. Fert. 13, 51.

FucHs, A. R. (1972) Uterine activity during and after mating in the rabbit. Fert. Steril, 23, 915.

GReENwald, G. S. (1956) Sperm transport in the reproductive tract of the female rabbit. Science, N.Y. 124, 586.

Hafez, E. S. E. \& Kanagawa, H. (1972) Giliated epithelium in the uterine cervix of the macaque and rabbit. 7 . Reprod. Fert. 28, 91.

Hamilton, G. E. (1951) Evidences of cyclic reproductive phenomena in the rabbit. Anat. Rec. 110, 553.

KRehiel, E. B., Lodge, J. R. \& Sharma, O. P. (1972) The effects of breeding stimuli on the rate of sperm transport in rabbits. F. Reprod. Fert. 29, 291.

Lightroot, R. J. \& Restall, B. J. (1971) Effects of site of insemination, sperm motility and genital tract contractions on transport of spermatozoa in the ewe. 7. Reprod. Fert. 26, 1.

Masters, W. H. \& Johnson, V. E. (1966) Human Sexual Response. Little Brown \& Co., Boston.

Mattner, P. E. (1966) Formation and retention of the spermatozoan reservoir in the cervix of the ruminant. Nature, Lond. 212, 1479.

MatTNer, P. E. (1968) The distribution of spermatozoa and leucocytes in the female genital tract in goats and cattle. F. Reprod. Fert. 17, 253.

Mattner, P. E. \& Braden, A. W. H. (1969) Comparison of the distribution of motile and immotile spermatozoa in the ovine cervix. Aust. F. biol. Sci. 22, 1069.

Morton, D. B. (1970) On the transport of spermatozoa in the female rabbit. Ph.D. thesis, University of Liverpool.

Nicholson, R. (1965) Vitality of spermatozoa in the endocervical canal. Fert. Steril. 16, 758.

Noyes, R. W., Adams, G. E. \& Walton, A. (1958) Transport of spermatozoa into the uterus of the rabbit. Fert. Steril. 9, 288.

PARKer, G. H. (1931) The passage of sperms and eggs through the oviducts in terrestrial vertebrates. Phil. Trans. R. Soc. B, 219, 381.

RIGBY, J. P. (1964) The fate of spermatozoa in the genital tract of the sow following artificial insemination. 5th Int. Congr. Anim. Reprod. \& A.I. Trento, 4, 421.

Skateine, P. N. \& Rumiantzewa, E. J. (1948) Speed of sperm transport in the female genital tract. Dokl. vses, Acad. sel'.-khoz. Nauk, 10, 45.

WeIsman, A. I. (1939) The endurance of spermatozoa within the vagina as compared with spermatozoal vitality outside of the body. Med. Rec., N.Y. 150, 87. 